\title{
SPM and phase-noise tolerant optical self-homodyne using a polarization-multiplexed and intensity-modulated pilot-carrier
}

\author{
Moriya Nakamuraa), Noriki Sumimoto, Yuya Takanashi, \\ and Ryoichiro Nakamura \\ School of Science and Technology, Meiji University, \\ 1-1-1 Higashimita, Tama-ku, Kawasaki, Kanagawa 214-8571, Japan
}

a)m_naka@meiji.ac.jp

\begin{abstract}
We propose a novel self-homodyne scheme using a polarizationmultiplexed and intensity-modulated pilot-carrier. The scheme has the capability to cancel nonlinear waveform distortion caused by self-phase modulation (SPM) in optical fibers, as well as the phase-noise of a laser diode. It has been known that a conventional constant-intensity pilot-carrier can cancel the waveform distortion caused by SPM to some extent. Theoretically, however, the proposed intensity-modulated pilot-carrier can completely cancel the effect of SPM. The performance in terms of the error vector magnitude was evaluated by numerical simulations of 50-km optical fiber transmission of 16-ary quadrature amplitude modulation (16QAM) signals.
\end{abstract}

Keywords: self-homodyne, pilot-carrier, SPM, phase-noise, optical fiber Classification: Fiber-Optic Transmission for Communications

\section{References}

[1] E. Ip and J. M. Kahn, "Compensation of dispersion and nonlinear impairments using digital backpropagation," J. Lightw. Technol., vol. 26, no. 20, pp. 34163425, Oct. 2008. DOI:10.1109/JLT.2008.927791

[2] L. Liu, L. Li, Y. Huang, K. Cui, Q. Xiong, F. N. Hauske, C. Xie, and Y. Cai, "Intrachannel nonlinearity compensation by inverse volterra series transfer function," J. Lightw. Technol., vol. 30, no. 3, pp. 310-316, Feb. 2012. DOI: 10.1109/JLT.2011.2182038

[3] X. Liu, A. R. Chraplyvy, P. J. Winzer, R. W. Tkach, and S. Chandrasekhar, "Phase-conjugated twin waves for communication beyond the Kerr nonlinearity limit," Nat. Photonics, vol. 7, pp. 560-568, May 2013. DOI:10.1038/ nphoton.2013.109

[4] T. Sakamoto, G.-W. Lu, and T. Kawanishi, "Oppositely-biased dual-polarization IQ modulators for nonlinearity-tolerant polarization-multiplexed phaseconjugated twin-signals generation," European Conference on Optical Communication (ECOC2015), Th.1.4.6, Sept. 2015. DOI:10.1109/ECOC. 2015.7341854 
nonlinearities using conjugate repetition," Opt. Express, vol. 23, no. 3, pp. 2392-2402, Jan. 2015. DOI:10.1364/OE.23.002392

[6] T. Miyazaki, "Linewidth-tolerant QPSK homodyne transmission using a polarization-multiplexed pilot carrier," IEEE Photon. Technol. Lett., vol. 18, no. 2, pp. 388-390, Jan. 2006. DOI:10.1109/LPT.2005.862363

[7] M. Nakamura, Y. Kamio, and T. Miyazaki, "Linewidth-tolerant 10-Gbit/s 16-QAM transmission using a pilot-carrier based phase-noise cancelling technique," Opt. Express, vol. 16, no. 14, pp. 10611-10616, Jul. 2008. DOI:10.1364/OE.16.010611

[8] Y. Kamio, M. Nakamura, and T. Miyazaki, "SPM-tolerant 16QAM with homodyne detection using pilot-carrier-polarization-multiplexing," IEICE Technical Report, CS2009-70, Jan. 2010 (in Japanese).

[9] Y. Kamio, M. Nakamura, and T. Miyazaki, "Pilot-symbol aided homodyne detection for optical fiber transmission," IEICE Technical Report, CS2007-14, Jun. 2007 (in Japanese).

[10] N. Sumimoto, R. Nakamura, and M. Nakamura, "SPM and phase-noise compensation using a polarization-multiplexed and intensity-modulated pilotcarrier," Optoelectronics and Communications Conference (OECC2017), P3009, Aug. 2017. DOI:10.1109/OECC.2017.8114881

[11] G. P. Agrawal, Fiber-optic Communication Systems (Second edition), WileyInterscience, 1997.

\section{Introduction}

Waveform distortion caused by optical nonlinear effects, including self-phase modulation (SPM), is becoming a more serious problem in optical transmission systems, especially when using optical multilevel modulation schemes, such as 16-ary quadrature amplitude modulation (16QAM), because the signal power varies according to the transmitted symbols, resulting in a large peak-to-averagepower ratio (PAPR). Some methods have been proposed for compensating for nonlinear effects, including digital back propagation (DBP) and the Volterra series transfer function (VSTF) [1, 2]. However, these methods based on digital signal processing (DSP) need an enormous amount of calculations, which causes serious time delay and increase in cost at the receiver. Phase conjugate twin wave (PCTW) schemes are drawing attention as a new method that has the ability to cancel nonlinearities in optical domain [3]. In this scheme, a phase-conjugate lightwave is multiplexed with the modulated signal by polarization multiplexing or timedivision multiplexing (TDM) $[4,5]$. On the other hand, we have proposed and investigated novel schemes that have the ability to cancel SPM and phase noise at the same time. In these schemes, a pilot carrier with constant optical power is multiplexed with a modulated signal by polarization multiplexing or TDM $[6,7$, 8 , 9]. In this paper, we propose a novel pilot-carrier scheme. An intensitymodulated pilot-carrier is polarization-multiplexed with a modulated optical signal. The performance, in terms of the error vector magnitude (EVM), was investigated by numerical simulations. The results clarified that the SPM cancellation capability was improved compared to that achieved with conventional constant-intensity pilot-carrier schemes. 


\section{Principle}

Figs. 1(a) and (b) schematically show the principle of the proposed polarizationmultiplexing and intensity-modulated pilot-carrier [10]. The lightwave from a laser diode (LD) is split into two polarization components using a polarization beam splitter (PBS). One polarization component is modulated as a signal. On the other hand, only the intensity of the other component is modulated so that it has the same power as the signal component, as shown in Fig. 1(a). This intensity-modulated component is used as pilot-carrier, which provides an absolute optical phase reference for self-homodyne detection [7]. Fig. 1(b) shows the principle of SPM and phase-noise cancellation. The intensity-modulated pilot-carrier has the same amplitude as the modulated signal. As a result, the optical phases of the two polarization components are identically rotated by SPM, keeping the mutual relative phase angle between them. Furthermore, this relative phase angle does not fluctuate due to the phase-noise of the LD, because the pilot-carrier has the same phase-noise as the signal component. On the receiver side, self-homodyne detection is performed using the pilot-carrier as a phase reference, where the influence of SPM and phase-noise can be cancelled. The amplitude of the receiver signal after the self-homodyne varies according to the square of modulated optical signal. However, the influence can be cancelled by digital signal processing (DSP). In the case of the conventional constant-intensity pilot carrier, however, the intensity of the pilot carrier is not the same as the modulated signal because the

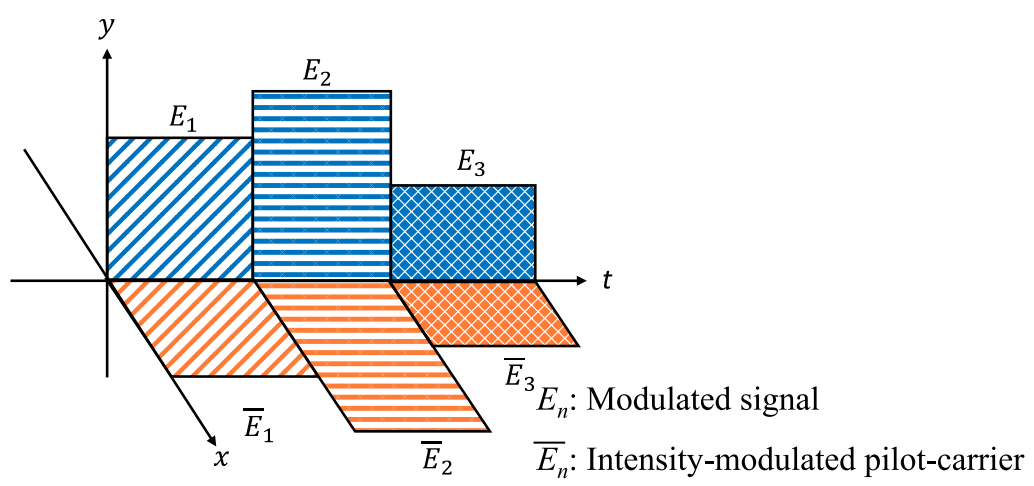

(a) Polarization-multiplexed and intensity-modulated pilot-carrier.

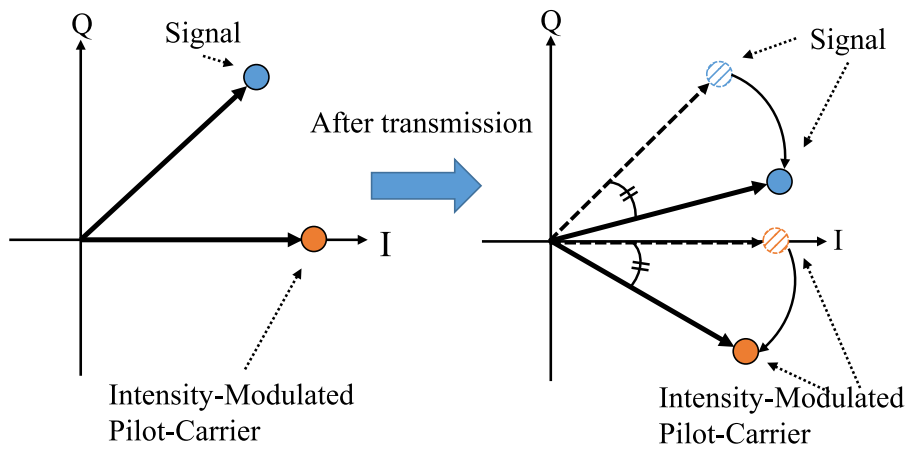

(b) Compensation of nonlinear distortion and phase-noise.

๑) IEICE 2019

DOI: 10.1587/comex.2019XBL0044 Received March 9, 2019 Accepted May 14, 2019

Publicized May 23, 2019

Copyedited August 1, 2019 


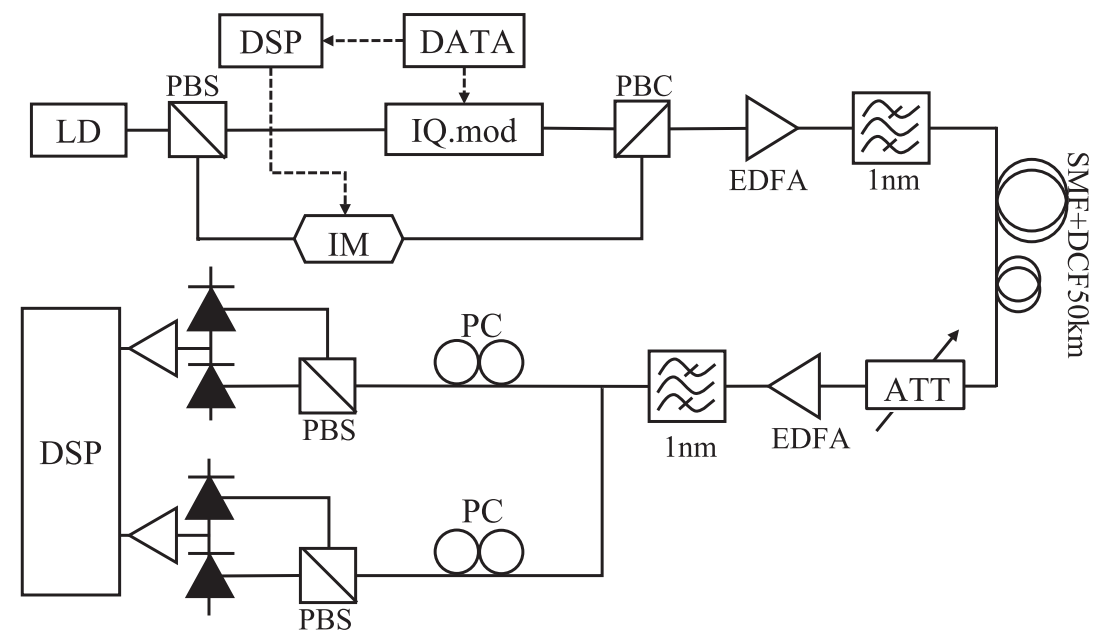

Fig. 2. System setup.

intensity of the modulated signal varies symbol by symbol. Therefore, the optical phases of the two polarization components are not identically rotated by SPM. Therefore, the conventional pilot carrier scheme can not keep the mutual relative phase angle between the modulated signal and the pilot carrier. This is the reason why the SPM-compensation capability of the conventional constant-intensity pilot carrier is limited [8].

\section{System setup}

The performance of the proposed scheme was evaluated by numerical simulation. Fig. 2 shows a 50-km 16QAM signal transmission system using a polarization multiplexed and intensity-modulated pilot-carrier and self-homodyne detection. The lightwave from an LD is split into two polarization components using a PBS. One polarization component is modulated by 10-GSymbol/s 16QAM with PRBS $2^{11}-1$ data, whereas the other component is intensity modulated so that the two polarization components have the same optical power. Here, we assumed that the electrical skew between the data signal and the pilot carrier is zero. It should be noted that this modulation can be realized using a commercially available dual-polarization quadrature phase-shift keying (DP-QPSK) modulator. The modulated lightwave is transmitted by a standard single-mode fiber (SSMF) and a dispersion compensating fiber (DCF) having a total length of $50 \mathrm{~km}$, thus canceling the total chromatic dispersion. The dispersion of the SSMF and the DCF was $16.75 \mathrm{ps} / \mathrm{nm} / \mathrm{km}$ and $-77 \mathrm{ps} / \mathrm{nm} / \mathrm{km}$, respectively. The noise figure of the Erdoped fiber amplifiers (EDFAs) was $3 \mathrm{~dB}$. The transmission through the optical fibers was numerically calculated using well-known split-step Fourier method. The input power to the optical fibers was $10 \mathrm{dBm}$. The linewidth of the LD was varied between $100 \mathrm{kHz}$ and $30 \mathrm{MHz}$ in order to confirm the phase-noise cancellation capability. It was assumed that the phase fluctuation was taken to be a Gaussian random process [11]. On the receiver side, self-homodyne detection was performed using the pilot-carrier as a phase reference with PBS and DSP. The thermal noise of the electric devices in the transmitter and the receiver was neglected. Using the 
same transmission system, we also performed conventional homodyne detection without the pilot-carrier for comparison. Here, we assumed an ideal local oscillator with an optical power of $0 \mathrm{dBm}$, which was ideally synchronized to the optical signal. We also assumed an ideal clock recovery both in the self-homodyne and the conventional homodyne receivers. The received signals were evaluated by EVM which is defined as signal fluctuation from the ideal symbol position. The definition of the EVM is shown as

$$
E V M=\frac{1}{N} \sum_{i=1}^{N} \frac{\left|\boldsymbol{E}_{i}-\hat{\boldsymbol{E}}_{i}\right|}{\left|\hat{\boldsymbol{E}}_{i}\right|} \times 100 \%,
$$

where $\boldsymbol{E}_{i}$ is the complex amplitude of the $i$-th transmitted symbol, $\hat{\boldsymbol{E}}_{i}$ is the ideal complex amplitude of the $i$-th transmitted symbol, and $N$ is the total number of the transmitted symbols. Therefore, the value of EVM includes the phase rotation by SPM and the symbol fluctuation caused by noise.

\section{Results and discussion}

Figs. 3(a) shows the constellation of the received signal using conventional homodyne detection without a pilot-carrier. The received optical power was $-15 \mathrm{dBm}$. Here, the received optical power means the averaged optical power including both of the modulated signal and the pilot carrier. The linewidth of the LD was $100 \mathrm{kHz}$. The 16QAM signal was seriously distorted by SPM. When we employed a conventional constant-intensity pilot-carrier, we could observed an SPM cancellation capability to some extent, as shown in Fig. 3(b). We observed some waveform distortion where outer symbols were slightly rotated clockwise. However, when we employed the proposed intensity-modulated pilot-carrier, the performance of the SPM cancellation was improved, as shown in Fig. 3(c). Fig. 3(d) shows EVM characteristics versus received optical power. In the case of homodyne detection, the EVM was larger than $33 \%$ due to the waveform distortion caused by SPM. When we employed the conventional constant-intensity pilot-carrier, an EVM value of less than $9 \%$ was achieved. In the case of our proposed intensity-modulated pilot-carrier, however, the EVM performance was improved by more than about $5 \%$ in comparison with the conventional intensityconstant pilot-carrier. Furthermore, even when we used an LD with a linewidth of as large as $30 \mathrm{MHz}$, the performance was not degraded seriously due to the phasenoise cancellation capability, as shown in Fig. 3(e). We plotted the EVM performance of the 16QAM signals versus the linewidth of the LD in Fig. 3(f). The EVM degradation at a linewidth of $30 \mathrm{MHz}$ was less than $1 \%$. The EVM values in Fig. 3(d) are different form that in our previous report [10]. In the previous report, we employed a dispersion shift fiber (DSF) and extremely large optical input power of $17 \mathrm{dBm}$. The EVM difference was caused mainly by this points in the system setup. 


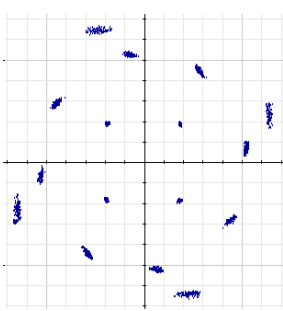

(a) Homodyne. (Linewidth: $100 \mathrm{kHz}$ )

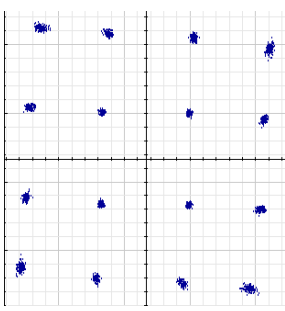

(b) Constant-intensity pilot-carrier.

(Linewidth: $100 \mathrm{kHz}$ )

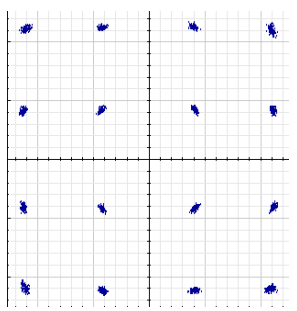

(c) Intensity-modulated pilot-carrier.

(Linewidth: $100 \mathrm{kHz}$ )

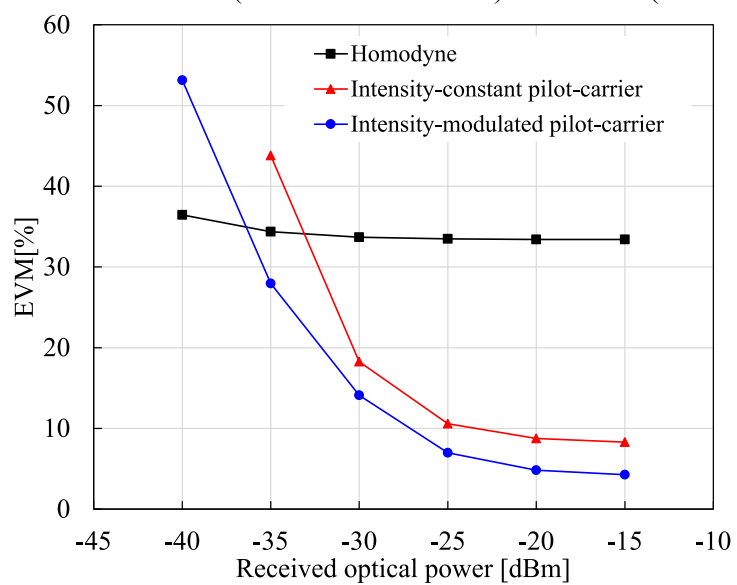

(d) EVM characteristics.

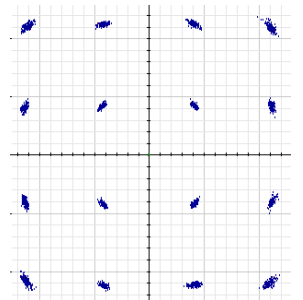

(e) Intensity-modulated pilot-carrier. (Linewidth: $30 \mathrm{MHz}$ )

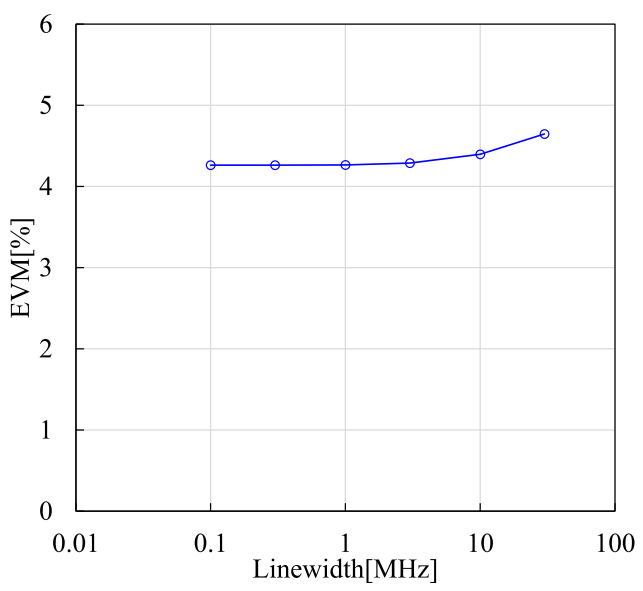

(f) EVM vs. linewidth characteristics.

Fig. 3. Results of numerical simulations.

\section{Conclusion}

A novel intensity-modulated pilot-carrier scheme was proposed. Numerical simulations clearly showed the SPM cancellation capability of the proposed scheme and improved performance over the conventional constant-intensity pilot-carrier scheme. Linewidth tolerant performance was also demonstrated in the simulation. The proposed scheme requires the construction of a special modulator; however, it can be implemented using a commercially available DP-QPSK modulator. 